\title{
Pregabalin versus gabapentin nel trattamento dei pazienti con neuropatia periferica: adattamento di un modello internazionale per la valutazione di costo/efficacia e costo/utilità alla realtà nazionale

Paolo Marchettini§, Lisa Da Deppo^, Lorenzo Pradelli*

\begin{abstract}
OBJECTIVE: To compare the economic impact of treating neuropathic pain with pregabalin versus gabapentin. DESIGN: A cost effectiveness analysis comparing costs and effects of pregabalin $375 \mathrm{mg} / \mathrm{die}$ versus gabapentin $1800 \mathrm{mg} /$ die in the perspective of the Italian National Healthcare Service was developed. The cost effectiveness analysis is examined alternatively in terms of the incremental cost per additional day with no or mild pain, and the incremental cost per quality-adjusted life-year (QALY) gained. Effects were derived from a pregabalin randomised clinical study 1008-155 and gabapentin 645-210 and 945-211 studies. Effects are expressed as reduction score of the VAS pain scale. Pharmacological costs were quantified according to the Italian market price of the drugs; healthcare procedure and hospitalisation costs were quantified on the basis of the National Tariff. Other healthcare services consumption data were derived from a Delphi Panel. To estimate the impact of pregabalin and gabapentin on daily pain experience in patients with neuropatic pain a Markov model is used. The dynamic simulation focuses on a hypothetical cohort of 1000 patients and simulates their daily pain experience over 12 weeks, to estimate clinical and economic outcomes for the group as a whole.

MAIN OUTCOME MEASURES AND RESULTS: The cost-effectiveness ratio for the use of pregabalin is less than 1 euro per additional day with no or mild pain and 468 euros per QALY. The sensitivity analysis conducted to examine the effects of decreasing gabapentin dose to $1200 \mathrm{mg} /$ die showes the consistency of the model results.

CONCLUSIONS AND RESULTS: Although pregabalin pure costs are higher than gabapentin costs, the analyses prove pregabalin to be more effective with a small additional cost per day with no or mild pain.
\end{abstract}

Keywords: pregabalin, gabapentin, neuropathic pain, cost effectiveness analysis

Farmeconomia e percorsi terapeutici 2005; 6 (3): 243-250

\section{INTRODUZIONE}

Il dolore neuropatico è definito come "dolore causato da lesioni primarie o disfunzioni del sistema nervoso" [1,2], è invalidante e con tendenza alla cronicizzazione. Il dolore neuropatico può essere classificato in centrale e periferico, in base all'eziologia del danno o alla sede anatomica [3].

I dolori neuropatici periferici più frequenti sono la neuropatia diabetica, la neuropatia posterpetica, la neuropatia correlata all'HIV, il dolore neuropatico correlato al cancro e il dolore a collo e schiena con componente neuropatica (radicolopatie). Sono considerati sindromi neuropatiche centrali la disestesia conseguente a lesioni del midollo spinale e dolore post infarto del tronco cerebrale o dell'encefalo [1,2].
La neuropatia diabetica rappresenta la causa di neuropatia periferica più frequente nel mondo occidentale. Nella neuropatia diabetica i sintomi variano da un lieve intorpidimento a un dolore profondo e lancinante oppure forte e incessante e possono essere estremamente fastidiosi alterando completamente la qualità di vita del paziente [4]. Uno studio statunitense ha evidenziato che circa due terzi dei pazienti diabetici presentano una neuropatia periferica [5]. Negli USA, il costo per il trattamento della neuropatia diabetica ammontava a circa 5-13 miliardi di dollari nel 2001, rappresentando fino al $27 \%$ del costo sanitario diretto sostenuto per il trattamento del diabete [6].
$\S$ Centro di Medicina del Dolore - Istituto Scientifico S. Raffaele, Milano

$\wedge$ Outcomes Research -

Pfizer Italia

* Centro di ricerche farmacoeconomiche Advanced Research Srl 
La neuropatia posterpetica è una complicanza comune dell'herpes zoster [7] che si presenta con un dolore intenso ed incessante e con un profondo impatto sulla qualità di vita e sullo stato funzionale [8].L'incidenza dell'herpes zoster aumenta con l'età dei soggetti [9]: varia tra 1,3 e 3,4 per 1000 pazienti/anno nella popolazione generale e tra 3,9 e 11,8 per 1000 pazienti/ anno nei soggetti con età superiore ai 60 anni. L'incidenza della neuropatia posterpetica varia tra il 9 e il $24 \%$ dei soggetti colpiti da herpes zoster [8] e la prevalenza aumenta all' aumentare dell'età: nei pazienti adulti con herpes zoster non adeguatamente trattato, la neuropatia posterpetica si manifesta circa nel $27 \%, 47 \%$ e $73 \%$ dei soggetti rispettivamente sopra i 55, 60 e 70 anni [10]. Uno studio inglese ha identificato il costo per tutta la durata della patologia di un paziente con neuropatia posterpetica in 770 sterline [11], mentre uno studio successivo, condotto negli Stati Uniti, ha rilevato che la spesa per il trattamento della neuropatia posterpetica varia da 34 a più di 150 dollari mensili per paziente [12], con un pesante impatto sulla qualità di vita [13].

La distinzione tra dolore neuropatico e altri tipi di dolore è importante, sia per la comprensione dell'eziopatogenesi sia ai fini del trattamento; il dolore neuropatico, infatti, risponde scarsamente ai farmaci analgesici normalmente utilizzati (oppioidi, antinfiammatori non steroidei, altri analgesici non oppioidi) per il controllo delle altre forme algiche. Ciò dipende in larga misura dal meccanismo sottostante la sensazione dolorosa che origina da un'ipereccitabilità neuronale nella sede della lesione. Coerentemente, i farmaci che mostrano la maggior efficacia nel controllo del dolore neuropatico sono gli antidepressivi triciclici (amitriptilina, nortriptilina) che aumentano la neurotrasmissione catecolaminergica e gli anticonvulsivanti (carbamazepina, gabapentin), che riducono l'eccitabilità neuronale [14].

Pregabalin è un nuovo analogo del GABA indicato per la terapia add-on dell'epilessia par- ziale e del dolore neuropatico periferico. Sebbene il pregabalin sia chimicamente simile al GABA, si ritiene che esplichi il suo effetto mediante l'attivazione della subunità alfa-2delta dei canali del $\mathrm{Ca}++$ potenziale-dipendenti. Come spesso accade con le terapie innovative, si tratta di un'opzione contemporaneamente più costosa ma più efficace dell'alternativa "standard" (gabapentin).

\section{OBIETTIVO}

Obiettivo del presente lavoro è la valutazione comparativa dell'impatto clinico ed economico di pregabalin e gabapentin nella terapia del dolore neuropatico periferico in pazienti affetti da nevralgia posterpetica e polineuropatia diabetica, mediante analisi di costo/efficacia e di costo/utilità incrementale.

\section{MATERIALIEMETODI}

Per l'esecuzione delle analisi, si è provveduto ad adattare al contesto sanitario nazionale un modello farmacoeconomico sviluppato da "The Neuropathic Pain Outcomes Modeling Working Group" [15].

\section{Il modello}

Il cuore del modello è un simulatore stocastico (metodo Montecarlo) in grado di stimare gli effetti del trattamento sull'intensità del dolore di una ipotetica coorte di pazienti con neuropatia periferica (Fig. 1), descritto nel dettaglio in una pubblicazione di Vera-Llonch et al [15].

In breve, a ogni paziente $(n=1000)$ viene assegnato un punteggio giornaliero medio da 0 a 10 (utilizzando una scala analogico visiva VAS) sulla base della distribuzione dei punteggi individuali pre-trattamento impostata per la coorte da analizzare. Nel secondo step, il simulatore genera una serie di punteggi giornalieri sulla base del punteggio medio assegnato e dell'assunta dispersione dei singoli valori intorno a tale media; infine vengono simulati gli effetti del trattamento, sulla base del-

\section{Figura1}

Modello di simulazione del dolore neuropatico [modificato da 14]

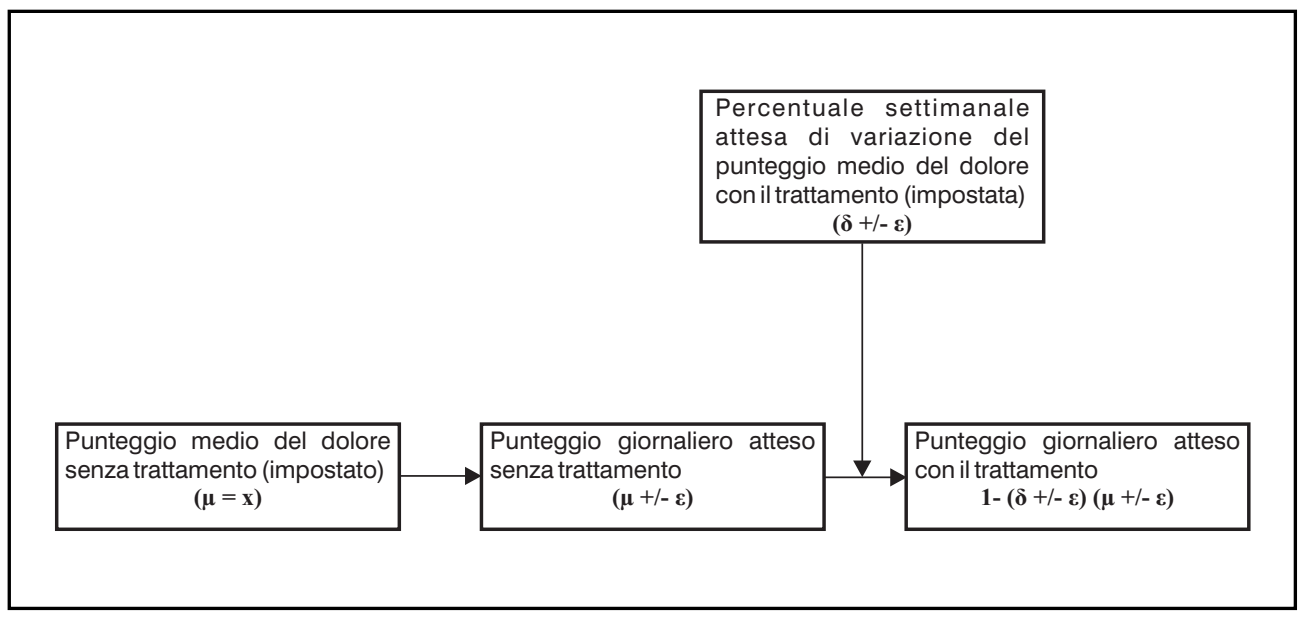




\begin{tabular}{cccc}
\hline Settimana & $\begin{array}{c}\text { Pregabalin } \\
\text { (dosaggio medio } \\
\mathbf{3 7 5} \mathbf{~ m g / d i e )}\end{array}$ & $\begin{array}{c}\text { Gabapentin } \\
\text { (dosaggio medio } \\
\mathbf{1 . 2 0 0} \mathbf{~ m g / d i e )}\end{array}$ & $\begin{array}{c}\text { Gabapentin } \\
\text { (dosaggio medio } \\
\mathbf{1 . 8 0 0} \mathbf{~ m g / d i e )}\end{array}$ \\
\hline 3 & $29,9 \%$ & $26,5 \%$ & $28,4 \%$ \\
6 & $44,2 \%$ & $31,6 \%$ & $33,6 \%$ \\
9 & $49,8 \%$ & $33,6 \%$ & $35,5 \%$ \\
12 & $54,1 \%$ & $34,3 \%$ & $36,2 \%$ \\
\hline
\end{tabular}

Tabella I

Riduzione percentuale dell'intensità media del dolore settimanale con $i$ trattamenti considerati la riduzione percentuale attesa del dolore, sempre tendendo in considerazione l'intera distribuzione dei valori di riduzione attesa. Questo processo viene ripetuto sulla coorte per 200 replicazioni, permettendo di calcolare valori medi, errore standard della media (ES) e intervalli di confidenza al 95\% (CI), espressi come numero totale di giorni trascorsi dalla coorte nei diversi stati di salute considerati (dolore assente o lieve - punteggio $<4$-, dolore moderato - punteggio $\geq 4$ e $<7$ - e dolore grave punteggio $\geq 7$ ) o con riduzioni significative ( $\geq$ $30 \%$ e $\geq 50 \%$ ) del dolore rispetto al baseline. Vari studi hanno dimostrato l'esistenza di una stretta correlazione tra questo punteggio e la qualità di vita percepita dai pazienti con dolore neuropatico $[16,17,18]$.

La valutazione economica delle terapie alternative si basa sulla quantificazione dei soli costi sanitari diretti, secondo la prospettiva del terzo pagante (SSN), ed è effettuata associando differenti livelli di consumo di risorse sanitarie ai diversi stati di salute considerati. Ad ognuna delle tipologie di risorsa considerata (visite mediche, test diagnostici e terapie diverse da quelle in studio) viene associata una probabilità che è funzione dell'intensità media del dolore nella settimana precedente. Il totale delle risorse consumate dalla coorte, nel corso del periodo di valutazione, viene quindi valorizzato in base alle tariffe e ai prezzi vigenti. Il medesimo approccio viene utilizzato per ricavare l'utilità associata alle strategie alternative: ogni stato di salute è associato a un differente livello di utilità ricavato da dati pubblicati o noti al decisore (da 0 a 1 corrispondenti agli estremi morte e miglior stato di salute immaginabile, rispettivamente). È dunque possibile stimare gli outcomes clinici ed economici di pazienti con neuropatia periferica conoscendone il livello medio di dolore prima del trattamento e la sua distribuzione nel campione, nonché i parametri economici locali e le funzioni di efficacia dei trattamenti (pregabalin e gabapentin). Queste ultime sono già incorporate nel modello per un orizzonte temporale di 12 settimane, corrispondente alla durata degli studi clinici facenti parte del programma di sviluppo del pregabalin. L'efficacia del pregabalin utilizzata per alimentare il modello è ricavata dalla riduzione media settimanale del punteggio alla VAS del dolore, rispetto al basale, osservata in uno studio clinico randomizzato e controllato versus placebo [19], condotto su pazienti con dolore neuropatico da polineuropatia diabetica (DPN) e nevralgia posterpetica $(\mathrm{PHN})$. La riduzione settimanale dello score utilizzata per il modello (Tabella I) è stata ottenuta nel ramo a dosaggio flessibile dello studio (scelto perché più prossimo alla prassi clinica e dunque più rilevante dal punto di vista economico, rispetto al ramo a dosaggio fisso), con un'esposizione di 12 settimane alla dose giornaliera media di $375 \mathrm{mg}$ (dose media di mantenimento $457 \mathrm{mg}$ ).

La funzione di efficacia del gabapentin adottata nel modello è ricavata dall'elaborazione dei dati ottenuti in due studi clinici randomizzati, il primo su pazienti con PHN [20] e il secondo su pazienti con DPN [21]. La dose media giornaliera nei due studi è stata di $2400 \mathrm{mg} / \mathrm{die}$; questo dosaggio, che rientra nella posologia di gabapentin, è al di sopra del dosaggio utilizzato nella normale pratica clinica in Italia. I dati di efficacia di gabapentin a dosi minori di $2400 \mathrm{mg} /$ die sono stati ricavati da un modello dose-risposta che è funzione del tempo trascorso [22].

Tale modello fornisce una stima delle riduzioni percentuali del dolore settimanale medio per ogni singola posologia giornaliera di gabapentin, nell'intervallo $600-3600 \mathrm{mg} / \mathrm{die}$, anche superato il limite temporale degli studi su cui si basa ( 8 settimane). In Tabella I presentiamo tali funzioni per i livelli dose che sono stati utilizzati per la valutazione economica (1200 e $1800 \mathrm{mg} / \mathrm{die})$.

Il beneficio clinico considerato dal modello è relativo alla sola terapia farmacologica; non viene attribuito alcun beneficio alle altre risorse sanitarie consumate dal paziente.

Il modello considera le seguenti assunzioni:

- che tutti i pazienti siano affetti da PHN o DPN e che vengano inizialmente gestiti dal MMG;

- che il livello medio pre-trattamento di intensità del dolore vari da moderato a severo (score $\geq 4$ ), e abbia una distribuzione simile a quella dei pazienti inclusi nello studio 1008-155 (Tabella II);

- che il dolore non sia correggibile mediante terapia della patologia di base (in altri termini, si suppone che i pazienti diabetici abbiano un adeguato controllo glicemico e i 


\begin{tabular}{lcc}
\hline Stato di salute & Punteggio giornaliero medio & \% Pazienti \\
\hline Dolore moderato & $4,0-<5,0$ & 8 \\
$5,0-<6,0$ & 19 \\
$6,0-<7,0$ & 18 \\
& $7,0-<8,0$ & 28 \\
Dolore grave & $8,0-<9,0$ & 19 \\
& $9,0-10,0$ & 8 \\
\hline
\end{tabular}

Tabella II

Distribuzione dell'intensità basale giornaliera media del dolore tra i pazienti con dolore neuropatico moderato-severo

pazienti con $\mathrm{PHN}$ non abbiano infezione in fase attiva);

- che, senza trattamento, l'intensità media del dolore rimarrebbe invariata nel corso delle 12 settimane;

- che non vi siano interruzioni del trattamento per inefficacia, eventi avversi o terapia alternativa (nerve block, neurostimolatore spinale, infiltrazioni, ecc.).

\section{L'adattamento}

L'adattamento del modello al contesto sanitario italiano ha permesso di sviluppare un' analisi costo/efficacia e costo/utilità. Ai vari stati di salute è stato assegnato un livello di utilità, il consumo di risorse è stato adeguato ai pattern clinici nazionali e valorizzato in base ai prezzi e alle tariffe vigenti, secondo la prospettiva del SSN (Tabella III).

I dati relativi all'utilità media dei vari stati di salute $(0,67$ per il dolore lieve, 0,46 per il dolore moderato e 0,16 per il dolore grave) sono stati ricavati dai risultati di un'indagine internazionale condotta su pazienti con dolore neuropatico arruolati in sei grandi nazioni europee (Francia, Germania, Italia, Regno Unito, Spagna e Paesi Bassi), in cui è stata dimostrata l'esistenza di una stretta correlazione tra il livello d'intensità del dolore e la qualità di vita percepita dai pazienti con neuropatia periferica [23].

Per la stima del ricorso allo specialista, agli interventi diagnostici e alle terapie alternative in funzione del grado di intensità del dolore si è utilizzato il metodo Delphi modificato, che ha coinvolto un panel di esperti nazionali nella gestione di pazienti con dolore neuropatico e i cui risultati principali sono riassunti in Tabella IV, accanto ai costi unitari delle risorse, valorizzati in base al tariffario per le prestazioni di assistenza specialistica (DM 22 luglio 1996), ai DRG nazionali e all'Informatore Farmaceutico 2005. Per il prezzo di pregabalin, è stato adottato quello della confezione da $150 \mathrm{mg}$ e per gabapentin la confezione da $300 \mathrm{mg}$. Fa eccezione, per tipologia di costo (costo-opportuni- tà invece che tariffa) e fonte dei dati (attualizzazione di un dato pubblicato), il valore attribuito alla visita presso il MMG [24].

\section{RISULTATI}

I risultati clinici ed economici della simulazione effettuata dal modello sono presentati come valori medi per paziente $+/$ - errore standard (ES).

L'analisi farmacoeconomica si basa sui rapporti costo/efficacia e costo/utilità incrementali. In particolare, il parametro di efficacia adottato è il numero di giorni con dolore assente o lieve, misura che connota il successo clinico nella gestione del dolore neuropatico periferico, mentre il parametro di utilità impiegato è il QALY (quality adjusted life-year). All'inizio della simulazione tale valore è ovviamente uguale a 0 in entrambi i gruppi, visto il livello d'intensità del dolore dei pazienti inclusi nello studio.

\section{SCENARIO BASE}

La scelta della posologia da valorizzare è un momento critico dell'analisi.

Poiché nel modello è stata presa in considerazione una coorte di pazienti molto gravi, per pregabalin la dose media valorizzata è stata di $375 \mathrm{mg} /$ die, mentre per gabapentin la dose media utilizzata per trattare questi pazienti in Italia è stata indicata in $1800 \mathrm{mg} /$ die. Lo scenario principale dell'analisi ha dunque confrontato gli outcomes a 12 settimane di pregabalin $375 \mathrm{mg} / \mathrm{die}$ e gabapentin $1800 \mathrm{mg} / \mathrm{die}$ e ha considerato sia $\mathrm{i}$ costi farmaceutici, sia quelli delle altre risorse sanitarie consumate, in accordo alle stime e ai valori monetari descritti nella sezione precedente.

Come indicato in Tabella IV, la terapia con pregabalin (PGB) è risultata essere associata $\mathrm{a}$ esiti clinici attesi migliori di quelli attesi con gabapentin (GBP) $1800 \mathrm{mg} /$ die; in particolare, nel corso delle 12 settimane dello scenario, i pazienti assegnati a PGB trascorrono mediamente 36 giorni con dolore lieve o assente, contro i 26 dei pazienti assegnati a GBP. Anche il numero di giorni con una riduzione clinicamente significativa del dolore (> 30\% e > 50\%) è mediamente superiore con PGB. Questo vantaggio comporta un guadagno, seppur lieve, anche nel numero di QALY (0,0092). La Tabella V riporta l'analisi dei costi sostenuti per le risorse sanitarie utilizzate secondo lo scenario principale.

L'analisi evidenzia che la terapia con pregabalin, al dosaggio medio giornaliero di $375 \mathrm{mg} / \mathrm{die}$, comporta un costo complessivo medio atteso superiore di soli 4,10 euro rispetto a gabapentin $1800 \mathrm{mg} /$ die per tutto il periodo di 12 settimane di trattamento. È utile prendere in considerazione anche i soli costi farmaceutici: in quest'ottica, il trattamento con pregabalin $375 \mathrm{mg} /$ die è associato a un costo medio superiore di 113 euro rispetto a gabapentin $1800 \mathrm{mg} /$ die per tutto il periodo di 12 settimane di trattamento. 
La Tabella VI riporta i rapporti costo/efficacia e costo/utilità incrementali, calcolati sia in base al costo sanitario totale, sia in base al solo costo farmaceutico.

Come evidenziato in tabella, il modello stima che il trattamento con pregabalin $375 \mathrm{mg}$ / die fa guadagnare un giorno senza dolore o con dolore lieve a un costo di 0,41 euro se nell' analisi vengono inclusi i costi sanitari indotti dalla gestione dei pazienti con neuropatia periferica. Se si considerano solo i costi farmaceutici, il risultato si traduce in un costo di 11

\begin{tabular}{|c|c|c|c|c|}
\hline \multirow{2}{*}{ Prestazione } & \multicolumn{3}{|c|}{ Probabilità di ricorso (\% di pazienti) } & \multirow{2}{*}{$\begin{array}{l}\text { Costo } \\
\text { (euro) }\end{array}$} \\
\hline & Dolore moderato & Dolore grave & Pazienti inviati allo specialista & \\
\hline \multicolumn{5}{|l|}{ Farmaci } \\
\hline Pregabalin 375 mg/die/pz & - & - & - & 4,13 \\
\hline Gabapentin 1.200 mg/die/pz & - & - & - & 1,53 \\
\hline Gabapentin 1.800 mg/die/pz & - & - & - & 2,78 \\
\hline \multicolumn{5}{|l|}{ Visite } \\
\hline Visita MMG & 10 & 20 & - & 13,28 \\
\hline $\begin{array}{l}\text { Visita Specialista (codice } 89.13 \text { ) } \\
\text { (media di } 3 \text { visite in } 12 \text { settimane } \\
\text { per paziente inviato) }\end{array}$ & 30 & 55 & - & 20,66 \\
\hline \multicolumn{5}{|l|}{ Procedure Diagnostiche } \\
\hline $\begin{array}{l}\text { Tomografia computerizzata del } \\
\text { rachide e dello speco vertebrale } \\
(88.38 .2)\end{array}$ & - & - & 36 & 137,89 \\
\hline Risonanza magnetica (88.93.1) & - & - & 46 & 241,70 \\
\hline $\begin{array}{l}\text { Test di velocità di conduzione } \\
\text { nervosa motoria (93.09.2) }\end{array}$ & - & - & 46 & 10,33 \\
\hline $\begin{array}{l}\text { Test di velocità di conduzione } \\
\text { nervosa sensitiva (93.09.1) }\end{array}$ & - & - & 35 & 10,33 \\
\hline $\begin{array}{l}\text { EcoDoppler degli arti superiori o } \\
\text { inferiori (88.77.2) }\end{array}$ & - & - & 31 & 43,90 \\
\hline Elettromiografia (93.08.3) & - & - & 50 & 10,33 \\
\hline \multicolumn{5}{|l|}{ Altre terapie } \\
\hline Terapia fisica (10 sessioni) (93.11.5) & - & - & 22 & 49,60 \\
\hline Farmaco infiltrazione $(04.81 .1)$ & - & - & 26 & 51,65 \\
\hline Nerve block (05.31) & - & - & 13 & 77,47 \\
\hline $\begin{array}{l}\text { Elettroterapia antalgica (10 sessioni) } \\
(93.39 .5)\end{array}$ & - & - & 18 & 30,50 \\
\hline $\begin{array}{l}\text { Impianto di stimolatore spinale } \\
\text { (DRG 004) }\end{array}$ & - & - & 21 & $6.430,40$ \\
\hline
\end{tabular}

Tabella III

Probabilità e costi delle risorse sanitarie considerate

\begin{tabular}{cccc}
\hline & Pregabalin & Gabapentin & Differenza \\
Parametro & (dosaggio medio & (dosaggio medio & (PGB-GPB) \\
& $375 \mathrm{mg} / \mathrm{die})$ & $1.800 \mathrm{mg} / \mathrm{die})$ & (1) \\
\hline
\end{tabular}

\section{Punteggio dolore (media, ES)}

$\begin{array}{llll}\text { Basale } & 7,0(0,0) & 7,0(0,0) & 0,0(0,0) \\ \text { Fine-trattamento } & 4,1(0,0) & 4,8(0,0) & -0,7(0,0)\end{array}$

Giorni (media, ES)

$\begin{array}{lccc}\text { Assenza o dolore lieve } & 36(0,3) & 26(0,3) & 10(0,4) \\ \text { Dolore moderato } & 32(0,3) & 38(0,3) & -7(0,4) \\ \text { Dolore severo } & 16(0,2) & 20(0,2) & -4(0,3) \\ >30 \% \text { riduzione punteggio VAS dolore } & 51(0,3) & 42(0,3) & 8(0,5) \\ >50 \% \text { riduzione punteggio VAS dolore } & 37(0,3) & 26(0,3) & 11(0,5) \\ \text { QALY(media, ES) } & 0,1142(0,0003) & 0,1050(0,0003) & 0,0092(0,0005)\end{array}$

Tabella IV

Outcomes clinici attesi secondo lo scenario base 
Tabella V

Analisi dei costi sostenuti per le risorse sanitarie utilizzate secondo lo scenario principale

\begin{tabular}{lccc}
\hline \multicolumn{1}{c}{ Costi } & $\begin{array}{c}\text { Pregabalin } \\
\text { (dosaggio medio } \\
\mathbf{3 7 5} \mathbf{~ m g / d i e )}\end{array}$ & $\begin{array}{c}\text { Gabapentin } \\
\text { (dosaggio medio } \\
\mathbf{1 . 8 0 0 ~} \mathbf{~ m g / d i e )}\end{array}$ & $\begin{array}{c}\text { Differenza } \\
\text { (PGB-GPB) }\end{array}$ \\
\hline Farmaco & $346,92(0,00)$ & $233,52(0,00)$ & $113,40(0,00)$ \\
Visite MMG & $9,52(0,45)$ & $11,71(0,51)$ & $-2,19(0,71)$ \\
Visite specialista & $8,76(0,31)$ & $10,28(0,33)$ & $-1,52(0,42)$ \\
Test diagnostici & $78,09(3,69)$ & $91,74(4,34)$ & $-13,65(5,63)$ \\
Terapie alternative & $584,12(61,68)$ & $676,06(61,92)$ & $-91,94(86,45)$ \\
Totale & $1.027,40(62,49)$ & $1.023,30(63,15)$ & $4,10(87,72)$ \\
\hline
\end{tabular}

euro per giorno senza dolore o con dolore lieve. Questi valori, nell' analisi costo-utilità, sono rispettivamente di circa 468 e 12.805 euro per QALY guadagnato, a seconda che si consideri il costo sanitario complessivo ed il solo costo farmaceutico, rispettivamente.

\section{Analisi di sensibilità}

La funzione di efficacia del gabapentin, calcolata dal modello di dose-risposta, risulta essere piuttosto piatta, poiché all'aumentare del dosaggio giornaliero corrispondono incrementi molto lievi di efficacia. Per verificare la sensibilità delle stime del modello alle variazioni del dosaggio di gabapentin, è stata ripetuta l'intera simulazione utilizzando il dosaggio di $1200 \mathrm{mg} /$ die per gabapentin, mantenendo costante il dosaggio di pregabalin. Le Tabelle VII, VIII e IX mostrano i risultati di quest'analisi.

Il modello si rivela piuttosto sensibile alla variazione di questo parametro: di fronte a una sostanziale equivalenza dei risultati terapeutici rispetto al dosaggio superiore, la posologia di $1200 \mathrm{mg} /$ die induce costi significativamente in-

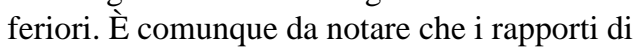
costo/utilità calcolati prendendo in considerazione sia il complesso dei costi sanitari associati alle due terapie, sia i soli costi farmaceutici rimangono ampiamente entro la soglia della disponibilità a pagare delle società industria-

PGB 375 mg/die vs GBP 1.800 mg/die (media, IC 95\%)

$\begin{array}{lc}\text { Costi totali } & \\ \text { Euro/giorno con dolore } & 0,41 \text { (dominante; 16) } \\ \text { assente o lieve guadagnato } & 468 \text { (dominante; 17.639) } \\ \text { Euro/QALY guadagnato } & \\ \text { Costi farmaceutici } & 11(10 ; 12) \\ \text { Euro/giorno con dolore } & 12.805(11.606 ; 14.134) \\ \text { assente o lieve guadagnato } & \\ \text { Euro/QALY guadagnato } & \end{array}$

Tabella V

Costo-efficacia e costo/utilità incrementali stimate per PGB 375 mg/die vs GBP $1800 \mathrm{mg} / \mathrm{die}$ lizzate, convenzionalmente fissato in circa 50.000 euro [25].

\section{DISCUSSIONE E CONCLUSIONI}

Per fornire una valutazione farmacoeconomica, in assenza di studi osservazionali di confronto diretto delle implicazioni cliniche ed economiche dell'utilizzo di due o più opzioni terapeutiche, è necessario ricorrere a modelli matematici in grado di stimare gli esiti sulla base delle migliori evidenze disponibili.

La qualità di ogni modello farmacoeconomico, oltre che dalle caratteristiche intrinseche (metodologia appropriata, fedeltà del percorso terapeutico riprodotto, esattezza della stima dei costi, inclusione delle misure di incertezza, ecc.) dipende strettamente dalla qualità dei dati clinici su cui è basato. I dati clinici per il confronto pregabalin vs gabapentin nel trattamento del dolore neuropatico sono ottenuti indirettamente da studi pubblicati che hanno confrontato ciascun farmaco con placebo. Questo approccio non permette di escludere che vi siano fattori potenzialmente confondenti (caratteristiche cliniche dei pazienti, percorsi diagnostico-terapeutici differenti) che contribuiscono alle differenze di efficacia osservate. Tuttavia, un attento esame dei dati riportati dagli studi utilizzati sembra escludere differenze eclatanti di disegno o di campionamento. Un secondo aspetto cruciale della presente analisi, strettamente correlato al precedente, è la funzione di efficacia utilizzata per gabapentin, ricavata da una rielaborazione matematica di dati ottenuti in studi differenti e a vari dosaggi. Osservando in maniera acritica le riduzioni percentuali dal dolore in relazione alle varie posologie calcolate dal modello dose-risposta, l'incremento di efficacia ottenibile con l'aumento di dosaggio appare molto basso. I valori riportati sono espressione di efficacie medie, mentre la posologia del farmaco è altamente individualizzata, nel senso che per il singolo paziente va ricercata la dose che conferisce il maggior beneficio clinico ottenibile. La pratica clinica indica che il dosaggio più comunemente associato all'efficacia e tollerabilità desiderabili è di 1800 


\begin{tabular}{lccr}
\hline \multicolumn{1}{c}{ Parametro } & $\begin{array}{c}\text { Pregabalin } \\
\text { (dosaggio medio } \\
\mathbf{3 7 5} \mathbf{~ m g / d i e )}\end{array}$ & $\begin{array}{c}\text { Gabapentin } \\
\text { (dosaggio medio } \\
\mathbf{1 . 2 0 0} \mathbf{~ m g / d i e )}\end{array}$ & $\begin{array}{c}\text { Differenza } \\
\text { (PGB-GPB) }\end{array}$ \\
\hline $\begin{array}{l}\text { Punteggio dolore (media, ES) } \\
\text { Basale }\end{array}$ & $7,0(0,0)$ & $7,0(0,0)$ & $0,0(0,0)$ \\
Fine-trattamento & $4,1(0,0)$ & $4,9(0,0)$ & $-0,8(0,0)$ \\
Giorni (media, ES) & $37(0,3)$ & $24(0,3)$ & $12(0,4)$ \\
Assenza o dolore lieve & $32(0,3)$ & $40(0,3)$ & $-8(0,5)$ \\
Dolore moderato & $16(0,2)$ & $20(0,3)$ & $-4(0,4)$ \\
Dolore severo & $51(0,3)$ & $41(0,4)$ & $10(0,5)$ \\
$>$ 30\% riduzione punteggio VAS dolore & $37(0,3)$ & $24(0,3)$ & $13(0,5)$ \\
$>$ 50\% riduzione punteggio VAS dolore & $0,1144(0,0003)$ & $0,1040(0,0003)$ & $0,0104(0,0004)$ \\
\hline
\end{tabular}

\begin{tabular}{lccc}
\hline \multicolumn{1}{c}{ Costi } & $\begin{array}{c}\text { Pregabalin } \\
\text { (dosaggio medio } \\
\mathbf{3 7 5} \mathbf{~ m g / d i e )}\end{array}$ & $\begin{array}{c}\text { Gabapentin } \\
\text { (dosaggio medio } \\
\mathbf{1 . 2 0 0 ~} \mathbf{~ m g / d i e )}\end{array}$ & $\begin{array}{c}\text { Differenza } \\
\text { (PGB-GPB) }\end{array}$ \\
\hline Farmaco & $346,92(0,00)$ & $128,52(0,00)$ & $218,40(0,00)$ \\
Visite MMG & $9,43(0,45)$ & $11,99(0,51)$ & $-2,56(0,71)$ \\
Visite specialista & $8,78(0,30)$ & $10,34(0,33)$ & $-1,56(0,44)$ \\
Test diagnostici & $78,34(4,01)$ & $92,60(4,71)$ & $-14,26(6,02)$ \\
Terapie alternative & $584,22(51,98)$ & $684,07(64,59)$ & $-99,85(85,40)$ \\
Totale & $1.027,70(53,86)$ & $927,52(66,07)$ & $100,18(87,64)$ \\
\hline
\end{tabular}

$\mathrm{mg} /$ die. Un altro limite del modello è rappresentato dall'inclusione di interventi diagnostici e terapeutici cui vengono associati solamente costi e nessun beneficio clinico. Trattandosi di risorse il cui consumo è funzione del grado di intensità del dolore, questo approccio risulta sfavorire il farmaco meno efficace, cioè il gabapentin. Una tale assunzione si è resa necessaria per la mancanza di dati sul beneficio associato a queste terapie, in particolare se utilizzate in concomitanza ai farmaci.

D'altro canto, il trattamento con pregabalin è associato a notevoli vantaggi clinici rispetto al gabapentin, che non sono presi in considerazione dal modello [22].

Pregabalin può essere somministrato anche due volte al giorno, aumentando così la compliance del paziente. Dal punto di vista farmacocinetico, pregabalin presenta un assorbimento rapido e raggiunge livelli plasmatici costanti in 1-2 giorni, con una correlazione lineare tra dose e concentrazione plasmatica che permette un aggiustamento del dosaggio rapido e prevedibile.

Inoltre pregabalin viene impiegato a dosaggi più bassi e può essere somministrato in associazione ad altri farmaci senza determinare interazioni clinicamente significative.

In sintesi si può quindi affermare che pregabalin presenta un efficace controllo del dolore neuropatico, con una rapida comparsa dell'effetto clinico e con eventi avversi lievimoderati e transitori.

In conclusione, pur considerando i limiti posti dal modello, i risultati della nostra analisi indicano che pregabalin è significativamente più efficace di gabapentin nel controllo del dolore neuropatico associato a DPN e PHN, e che i benefici clinici aggiuntivi ottenibili con il suo utilizzo si producono a un costo inferiore a quello fissato come limite della disponibilità a pagare delle società avanzate [25].
Tabella VII

Outcomes clinici attesi secondo l'analisi di sensibilità

\section{Tabella VIII}

Analisi dei costi sostenuti per le risorse sanitarie utilizzate secondo l'analisi di sensibilità

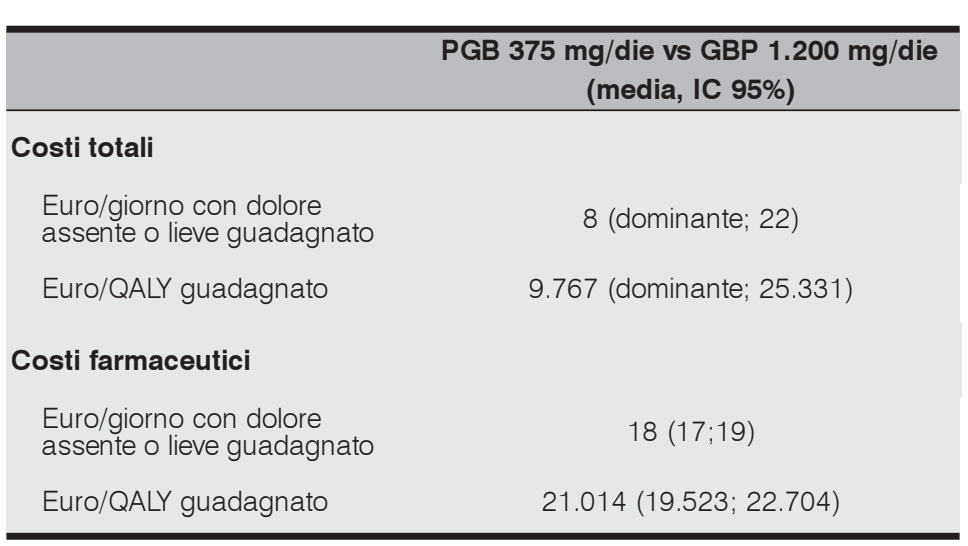

Tabella IX

Costo-efficacia e costo/utilità incrementali stimate per P $375 \mathrm{mg} /$ die vs $\mathrm{G}$ 1200 mg/die 


\section{BIBLIOGRAFIA}

1. Dworkin R. An overview of neurophatic pain: syndromes, symptoms, signs, and several machanisms. Clin J Pain 2002;18:343-9

2. Merskey H, Bogduk N. Classification of chronic pain: description of chronic pain syndromes and definitions of pains terms IASP press, Seattle 1994

3. Woolf CJ and Mannion RJ. Neuropathic pain: aetiology, symptoms, mechanisms, and management. Lancet 1999:353:1959-64.

4. Quattrini C and Tesfaye S. Understanding the impact of painful diabetic neuropathy. Diabetes Metab Res Rev 2003;19:S2-8

5. Dick PJ et al. The prevalence by staged severity of various types of diabetic neuropathy, retinophaty, and nephropathy in a population.based cohort: the Rochester Diabetic Neuropathy Study. Neurology 1993;43:817-824

6. Gordois A, Scuffham P, Shearer A, Oglesby A, Tobian JA. The health care costs of diabetic peripheral neuropathy in the US. Diabetes Care. 2003 Jun;26(6):1790-5.

7. Alper BS and Lewis PR. Treatment of postherpetic nevralgia: a systematic review of the literature. J Fam Pract 2002;51(2):121-128

8. Dworkin RH and Portenoy RK. Pain and its persistence in herpes zoster. Pain 1996;67:241-251

9. Ragazzino MW et al. Population-based study of herpes zoster and its sequalae. Medicine 1982;61(5):310-316

10. Kost RG and Strauss SE. Postherpetic neuralgia-pathogenesis, treatment, and prevention. N Engl J Med 1996;335(1):32-42

11. Davies L et al. The cost of treatment foe post-herpetic neuralgia in the UK. Pharmacoeconomics 1994;6(2):142-148

12. Smith KJ and Roberts MS. Antiviral therapies for herpes zoster infections. Are they economically justifiable? Pharmacoeconomics 2000;18(2):95-104

13 Oster G, Harding G, Dukes E, Edelsberg J, Cleary PD. Pain, medication use, and health-related quality of life in older persons with postherpetic neuralgia: results from a population-based survey. J Pain. 2005 Jun;6(6):356-63

14. Backonja MM. Use of anticonvulsants for treatment of neuropathic pain. Neurology. 2002 Sep 10;59(5 Suppl 2):S147.

15. Vera-Llonch M, Dukes E, Delea TE, Wang ST, Oster G; The Neuropathic Pain Outcomes Modeling Working Group. Treatment of peripheral neuropathic pain: A simulation model. Eur J Pain. 2005 Jun 22

16. Zelman DC, Dukes E, Brandenburg N, Bostrom A, Gore M. Identification of cut-points for mild, moderate and severe pain due to diabetic peripheral neuropathy.Pain. 2005 May;115(1-2):29-36.

17. Farrar JT, Young JP Jr, LaMoreaux L, Werth JL, Poole RM. Clinical importance of changes in chronic pain intensity measured on an 11-point numerical pain rating scale. Pain. 2001 Nov;94(2):149-58.

18. Galer BS, Gianas A, Jensen MP Painful diabetic polyneuropathy: epidemiology, pain description, and quality of life. Diabetes Res Clin Pract. 2000 Feb;47(2):123-8

19. Freynhagen $\mathrm{R}$ et al. Efficacy of pregabalin in neuropathic pain evaluated in a 12-week, randomised, double-blind, multicentre, placebo-controlled trial offlexible- and fixed-dose regimens. Pain. 2005 Jun;115(3):254-63

20. Rowbotham M, Harden N, Stacey B, Bernstein P, Magnus-Miller L. Gabapentin for the treatment of postherpetic neuralgia: a randomized controlled trial. JAMA. 1998 Dec 2;280(21):1837-42.

21. Backonja M, Beydoun A, Edwards KR, Schwartz SL, Fonseca V, Hes M, LaMoreaux L, Garofalo E. Gabapentin for the symptomatic treatment of painful neuropathy in patients with diabetes mellitus: a randomized controlled trial. JAMA. 1998 Dec 2;280(21):1831-6

22. Wesche D and Bockbrader H. A pharmacokinetic comparison of pregabalin and gabapentin. The Journal of Pain 5(3) Supplement 1 S29. 2005 Annual American Pain Society Meeting

23. McDermott AM, Toelle TR, Rowbotham DJ, Schaefer CP, Dukes EM. The burden of neuropathic pain: results from a cross-sectional survey. European Journal of Pain, article in press

24. Lucioni C, Garancini MP, Massi-Benedetti M, Mazzi S, Serra G; CODE-2 Italian Advisory Board. The costs of type 2 diabetes mellitus in Italy: a CODE-2 sub-study. Treat Endocrinol. 2003;2(2):121-33.

25. Barbieri M, Drummond M, Willke R, Chancellor J, Jolain B, Towse A. Variability of cost-effectiveness estimates for pharmaceuticals in Western Europe: lessons for inferring generalizability. Value Health. 2005 Jan-Feb;8(1):10-23. 